\title{
Gestión logística con enfoque en la ISO28000, para empresas comerciales.
}

\section{Logistic management system with ISO28000 focus for commercial companies.}

Diana Alexandra Rocano Criollo. ${ }^{1}$, Cecilia Ivonne Narváez Zurita. ${ }^{2}$, Juan Carlos Erazo Álvarez. ${ }^{3}$ y Kléber Antonio Luna Altamirano. ${ }^{4}$

\section{DOI: https://doi.org/10.33262/visionariodigital.v3i2.1.550}

\begin{abstract}
.
The success of any organization set up to put products on the market depends on its ability to achieve a successful combination of different factors like quantity, quality, opportunity, and the cost for goods and services that it provides. Against this backdrop, this research work aims to design a Logistics Management System for the Company Megalimpio Cía. Ltda. as means of ensuring appropriate control of all its operations and reduce possible risks in accordance with ISO 28000.

This article contains a detailed analysis of the specialized literature on the topic, results of the surveys, the Logistic Management System Proposal, research findings related to the main logistics activities within the company in which the study was carried out. The current research was performed for two years; also, it was approved by the company's board and had the support of workers involved in the supply chain. Consequently, there was required a comprehensive analysis of the

${ }^{1}$ Universidad católica de Cuenca, Posgradista Maestría en Contabilidad y Auditoría, darocanoc075@psg.ucacue.edu.ec

${ }^{2}$ Universidad Católica de Cuenca, Subdirección de Posgrado, Cuenca, Ecuador, inarvaez@ucacue.edu.ec

${ }^{3}$ Universidad Católica de Cuenca, Subdirección de Posgrado, Cuenca, Ecuador, jcerazo@ucacue.edu.ec

${ }^{4}$ Universidad católica de Cuenca, Subdirección de Posgrado, Cuenca, Ecuador, Universidad Católica de Cuenca,klunaa@ucacue.edu.ec
\end{abstract}


effectiveness in the logistics operation through surveys targeted to all the staff, indepth interviews with the company's executives, and finally, observation forms. After the diagnostic, the main conclusions that came out were the high prices due to an excessive amount of inventory, lack of control over inventory, and low profitability.

Keywords: Logistics, supply chain, internal control, inventory management, risks, ISO 28000.

\section{Resumen.}

El éxito de toda organización creada para comercializar productos en el mercado depende de la capacidad que tenga para lograr una conjugación exitosa de la cantidad, calidad, oportunidad y costo de los bienes y servicios que ofrece. Ante este escenario, la presente investigación tiene por finalidad diseñar un sistema de gestión logística con enfoque de la ISO 28000 para la empresa Megalimpio Cía. Ltda., que propicie un adecuado control de sus operaciones y mitigue los riesgos a los que se encuentra expuesta.

El artículo presenta el análisis de la literatura especializada en el tema, los resultados del diagnóstico, la propuesta del sistema de gestión logística y las conclusiones correspondientes a la investigación relacionadas con las actividades principales de la logística en la empresa objeto de estudio. La investigación se realizó durante dos años, fue aprobada por el directorio de la compañía y contó con la participación de todos los trabajadores involucrados en la cadena de suministro. Para tal efecto, se analizó íntegramente el funcionamiento de la operación logística mediante la aplicación de encuestas dirigidas al personal, entrevistas en profundidad dirigidas a los directivos y ficha de observación. Entre las principales conclusiones del diagnóstico se identificó los altos costos por mantener cantidades excesivas de inventario, deficiente control de inventarios y baja rentabilidad en la empresa.

Palabras claves: Logística, cadena de suministro, control interno, gestión de inventarios, riesgos, ISO 28000.

\section{Introducción.}

La gestión logística ha evolucionado a lo largo de la historia, se puede manifestar que tiene sus orígenes en el campo militar, en vista de que los soldados necesitaban buscar la forma 
de dotar, trasladar y almacenar eficientemente los recursos. La gestión logística tiene sus inicios militares a comienzos de la segunda guerra mundial en donde las tropas tenían que cumplir ciertos procesos para efectuar su labor de forma eficaz (Mora, 2016).

A mediados de los años sesenta, los empresarios empiezan a entender que la disminución de sus inventarios y el incremento de las cuentas por cobrar generaba un incremento de los flujos de caja y por tanto, vieron que la utilidad podía crecer si buscaban la manera de mejorar las operaciones de distribución de las mercancías (Manzur, 2014). Es así como para finales de los años ochenta surge un nuevo concepto de logística integrada, ésta hace referencia al conjunto de actividades que se relacionan a la distribución de los productos, incluyendo un sistema de información y control (Casanovas y Cuatrecasas, 2012).

En las últimas dos décadas, la gestión logística empresarial ha tomado posicionamiento, puesto que el mercado con el pasar de los años se han vuelto más exigente; la integración y la globalización en la actualidad obliga a las empresas a contender entre ellas y, la gestión logística busca el mejoramiento continuo para que la atención al cliente sea de calidad. Actualmente, la logística integral se ha transformado en un verdadero activo para las compañías ya que se puede medir la eficiencia y excelencia del servicio, hasta que el producto llegue a cada uno de sus clientes, siendo una herramienta competitiva que optimiza los recursos de la empresa en cada una de sus etapas (Mora, 2016).

La gestión logística se puede entender como un conjunto de actividades que organizan el flujo de bienes y servicios, desde su origen hasta la llegada al cliente, considerando el control y la gestión eficiente, con el fin de satisfacer los requerimientos del cliente (Gutiérrez y Prida, 1998) (Ballau, 2004) (Gibson, Mentzer, y Cook, 2005).

Dentro de los procedimientos logísticos, la cadena de suministro es un punto clave, debido a que de ésta depende el reabastecimiento óptimo y oportuno de los productos que la empresa requiere para su posterior comercialización, es importante tener claro el impacto económico de las diferentes fases que forman parte de la cadena de suministro, puesto que mediante su planificación, control y análisis es posible generar un ahorro (Mora y Martiliano, 2010).

Los procedimientos que intervienen en la cadena de suministros requieren del enlace de los subsistemas, técnicas y actividades referentes al movimiento del inventario con lo que la empresa cuenta para la distribución en todas sus etapas de comercialización, buscando alcanzar la satisfacción de los clientes y garantizar la sostenibilidad de las organizaciones (Pérez, 2006). 
$\mathrm{Al}$ respecto, Mora (2016) menciona que el objetivo de una gestión logística es aumentar las ventajas competitivas para generar un incremento en las utilidades, misma que deberá generarse con la comercialización del producto y para que este pueda llegar al cliente final se deberá contar con una buena gestión, por lo que se derivan los siguientes objetivos:

- Garantizar que el menor costo operativo sea un factor clave de éxito.

- Suministrar adecuada y oportunamente los productos al cliente.

- Transformar la logística en una característica que la diferencie ante los rivales.

Como soporte de la gestión logística se debe considerar el control en la logística, mismo que es un proceso en donde se compara el desempeño obtenido realmente, con el que se planteó como objetivo, lo que permite tomar decisiones o acciones correctivas para alcanzar los objetivos planteados. Este proceso consiste en la supervisión de las condiciones que pueden variar y tomar de manera anticipada las precauciones para lograr los resultados esperados (Ballau, 2004). Por consiguiente, la estructura de los procedimientos de control de entradas y salidas de la mercadería dentro de la organización deben basarse en estándares que se alineen con las metas que tenga en mente la gerencia, de la misma manera contar con personal calificado para la supervisión, para saber que se deben ajustar al presupuesto y tomar providencia en las decisiones empresariales e incrementar la rentabilidad. Por consiguiente, la administración debe realizar informes regulares en los que se pueda visualizar los costos, productividad del personal y de esta manera poder auditar de forma constante y así también permitir a la gerencia a tomar planes de contingencia a tiempo en caso de que se tenga que establecer ajustes.

En el sistema logístico la alta gerencia, así como el personal encargado busca controlar las acciones inherentes a los procedimientos de logística como son los de transporte, almacenamiento, inventario, el manejo del material y el procesamiento del pedido, para llevar un adecuado control mediante auditorias en los factores que están relacionados a la cadena de suministros.

Dentro de este sistema o proceso es necesario entender y gestionar las existencias, las mismas que se entienden como el inventario que dispone la empresa y se almacena en sus bodegas, esperando que dicho inventario pueda ser distribuido, vendido o consumido por los clientes y consumidores finales, donde la empresa debe conocer con exactitud el volumen de las entrada y salidas del inventario durante un periodo determinado, buscando en lo posterior solicitar a los proveedores los productos necesarios para su futura comercialización (Escudero, 2011). 
Las existencias varían por el consumo del producto es decir al momento que se realiza la venta del producto, dando lugar al requerimiento para la reposición de la mercancía, la rotación del inventario genera utilidad al momento de producirse la venta en los almacenes, lo que obliga a mantener en ellos una cantidad determinada, es decir, un inventario que haga referencia a un punto de equilibrio con cada producto ofertado, información que debe constar en los reportes (Martínez, 2001).

Para muchas empresas, es factible disminuir el inventario mejorando ciertos procesos en la programación, realizando estudios complementarios de tiempo y movimiento de inventarios. "El inventario es frecuentemente un síntoma o paliativo de áreas de oportunidad en la operación, un camino fácil pero costoso para obviar una tarea difícil que se encuentra latente en la empresa" (Martínez, 2001, p.58).

El inventario representa un uso importante en el canal de la cadena de suministros, por lo que se debe contar con políticas que consideren el análisis sobre la administración optima del inventario, contando con métodos que permitan el control eficiente y así la gerencia pueda determinar una planeación para poder realizar el presupuesto que necesita elaborar considerando todos los patrones que estarán relacionados para elaborar el informe.

Otro aspecto que hay que tomar en cuenta y enfocarse de la gestión logística son los denominados costos logísticos, los cuales permiten cuantificar en dinero los recursos a emplear en las diferentes actividades del proceso logístico, estos costos ayudan a explicar el beneficio sobre el capital invertido y la manera de como regresa la inversión a la empresa en un tiempo preestablecido.

La gestión de costos logísticos implica mantener un control y revisión exhaustiva para calcular la rentabilidad que genera el producto, costear apropiadamente y tomar decisiones sobre ese análisis. Una eficiente gestión permite la optimización de recursos en la cadena de suministros, pues un adecuado control ayuda a la gerencia tomar las decisiones relevantes en favor de la organización y sus beneficios. Se debe considerar que el colaborador encargado de la gestión logística debe presentar informes confiables y de ser el caso, tomar los correctivos pertinentes, mejorando los resultados de la gestión (Mora 2016).

La gestión de costos forma parte del ciclo que atraviesa la entrega del producto, donde primeramente es necesario conocer qué actividades se van a realizar dentro de todo el proceso de entrega de productos al cliente. Una vez se tenga el conocimiento preliminar, se debe buscar información acerca de los recursos necesarios para poder realizar esas acciones. 
Los procesos que la empresa ejecuta en referencia al abastecimiento y despacho de la mercadería, los costos financieros asociados al inventario, el transporte, los sistemas de control del inventario siendo indispensable incorporar un control de inventarios eficiente.

Existen dos sistemas que permiten llevar el sistema de registro de inventario: "el sistema periódico y el sistema permanente" (González, 2002, p.26). El sistema de inventarios periódico es un sistema de costos que faculta el control del inventario en un espacio de tiempo determinado, en el cual se debe paralizar las actividades, especialmente en el área de bodega para llevar a cabo el conteo físico del inventario que dispone la empresa en ese momento, para ello, la empresa establece en sus políticas cada qué tiempo se debe realizar este procedimiento. La empresa podrá determinar con exactitud su costo de venta real, cuando existe la toma física de los inventarios que en muchos de los casos se lo realizan al final del cierre contable. Para establecer el costo de ventas se toma el inventario inicial, se suman las adquisiciones del periodo y se resta el inventario final y dicho resultado constituye el costo de ventas generadas.

En el sistema de registro de inventario permanente, la empresa mantiene un registro actualizado del producto que forma parte del inventario de la empresa, por consiguiente, cuando se utiliza este sistema, es posible conocer con exactitud y en tiempo real el valor del inventario que se encuentra disponible. Cuando la empresa necesita consultar su inventario valorado "en ese momento se puede determinar el costo del inventario final y el costo de las mercancías vendidas directamente en sus propias cuentas dentro de la contabilidad de la empresa, sin tener que hacer un conteo físico del producto" (Gonzáles, 2002, p.28).

La ventaja que obtiene la empresa al utilizar este sistema es el ahorro de tiempo en el desarrollo del inventario, pues no se realiza el cierre de la empresa para el control de inventarios. Con este sistema se ejecutan inventarios permanentes con apoyo del personal encargado del inventario de la empresa, permitiendo obtener un resultado más exacto al momento del realizar el reconteo físico del inventario, de igual manera se puede identificar de manera oportuna las pérdidas o mermas del inventario que reposa en la bodega de la empresa frente al inventario que esta contabilizado.

Para optimizar los costos, se debe mantener un equilibrio con los costos de logística y el nivel de ventas. Entre los costos logísticos a considerar se tienen los costos de transporte como es el costo de operar con un trasporte propio, también el almacenamiento, costos que tiene que ver con el equipo de manejo de materiales, seguros reparación y depreciación del lugar físico donde se almacena el producto, personal que procesa las ordenes de pedido tanto a proveedores como el personal encargado de la parte operativa de la entrega del 
producto al cliente, costos relacionados a mantener un inventario equilibrado para poder ofertar al cliente (Mora y Martiliano, 2010).

El costo de pedido se determina en cualquier momento que se desarrolle el proceso de esta actividad en donde se considera el reabasteciendo del producto que fue comercializado, estos están vinculados con los costos de administrativos, los mismos que forman parte del proceso de la actividad de reabastecimiento del producto que se origina al momento en que se coloca la orden de pedido al proveedor y que el producto llega a las instalaciones de la compañía.

El transporte es una función de gran importancia en las actividades que se desarrollan en la gestión para que el producto llegue a su destino, la empresa trata de buscar de manera eficiente colocar el producto que la empresa oferta en el lugar de destino, es decir a manos de los clientes, de la misma manera busca que el producto llegue en las mejores condiciones y en el tiempo establecido (Anaya, 2007).

Figura 1. Indicadores de la gestión logística.

$\left.\begin{array}{c}\text { Orden de } \\ \text { Compra }\end{array}\right\rangle$ Transporte $\left.\left.\sum \begin{array}{c}\text { Recepción } \\ \text { e } \\ \text { inspeccion }\end{array}\right\rangle \begin{array}{c}\text { Colocación } \\ \text { del } \\ \text { Inventario }\end{array}\right\rangle$ Contabilización $\$ Auditoria $\left.\rangle \begin{array}{c}\text { Pago al } \\ \text { proveedor }\end{array}\right\rangle$

Fuente: Mora (2008)

Para una correcta planeación logística hay que considerar el pronóstico en el requerimiento del inventario, en esta etapa se determina la cantidad de recursos que se debe solicitar y que a su vez serán ejecutados para posteriormente determinar el recurso necesario para satisfacer los requerimientos del cliente. Gonzáles (2002) afirma: "los pronósticos permiten establecer la cantidad de producto a solicitar, la cantidad esperada de dinero a obtener por la venta del inventario, la cantidad de personal que se necesita para lograr atender todos los requerimientos de los clientes" (p.28).

Un pronóstico de ventas se entiende como la estimación de la venta del inventario bajo determinadas condiciones, dando como resultado crear condiciones que generan un nivel de ventas deseado, este pronóstico en su mayoría es elaborado por el gerente de ventas, para preparar dicho informe se consideraran varios parámetros como es el histórico de venta, ubicación geográfica, tipo de cliente, las acciones que posiblemente los competidores ejecuten en el mercado (Horngren, Sundem \& Stratton, 2007). 
Se puede decir entonces que "un pronóstico es la estimación de un acontecimiento futuro con datos del pasado, que utiliza la prospectiva, en la cual se partiendo de una serie de datos se formula una proyección para evaluar la ocurrencia probable de cualquier acontecimiento" (Martínez, 2001, p.56). Por lo tanto, los pronósticos se entienden como aquellos procesos que requieren de resultados garantizando que exista de por medio un trabajo complementario tanto en la planificación, logística y cadena de suministros para obtener los resultados esperados.

Otro tema de relevancia en la logística son los indicadores, estos se pueden entender como una función que muestra expresamente el comportamiento o desempeño de los procesos que la compañía ejecuta como parte de las actividades que desarrolla, éste se compara con los diferentes procesos que la compañía realiza para detectar desviaciones positivas o negativas. Se debe considerar que los indicadores deben cumplir con ciertas características muy importantes:

- Mide cambios en diversas situaciones a lo largo del tiempo.

- Instrumento que permite evaluar el proceso que lleva a cabo la compañía.

- Instrumento que permite mejorar un resultado en proyectos de desarrollo.

Entre los principales indicadores se puede considerar: Indicadores de compra y abastecimiento: Calidad de pedido generado; Volumen de compra. De inventarios: Rotación de mercadería; Duración de inventario; Vejez de inventario; Valor económico de inventario. Indicadores de almacenamiento y bodegaje: Costo de unidad almacenada; Costo de unidad despachada. Indicadores de transporte y distribución: Costo de transporte vs ventas (Mora, 2008).

Es importante en este punto insertar y analizar a una de las herramientas de gran valor para elaborar un modelo de gestión logística que es la norma ISO 28000, la cual es una especificación de un sistema de gestión que ofrece un modelo para todos los tipos y tamaños de organizaciones que operan o dependen de cualquier aspecto de la cadena de suministro, la misma fue desarrollada con la finalidad de crear un sistema de gestión de calidad, ambiental y de seguridad de la cadena de suministro (SCP Supply Chain Professionals, 2018).

Esta norma es aplicable a todas las empresas, cualquiera fuera su tamaño, cuyos procesos están inmersos a la fabricación, compra, producción, servicio, almacenaje, transporte y/o en procesos de ventas en todas sus etapas; que requieren de un procedimiento de seguridad con normas internacionales que se deben procesar en cada fase de la cadena de suministro. El objetivo de norma ISO 28000 es facilitar procedimientos para desarrollar prácticas 
correctas que permitirán reducir los riesgos para el personal, esta a su vez se ocupa de la seguridad del inventario en los procedimientos logísticos, identificando las posibles amenazas como el fraude, de la misma manera se ocupa de disminuir las causas de las eventualidades de seguridad (Larrucea, 2015).

\section{Metodología.}

La investigación se enmarco en un diseño mixto con la finalidad de tener una perspectiva más amplia y profunda de la gestión logística, logrando aproximaciones cualitativas y cuantitativas. El enfoque cualitativo se empleó en el análisis de la literatura especializada, en las narrativas, símbolos y elementos visuales aplicados en la propuesta. En cuanto al enfoque cuantitativo el fenómeno en estudio se representó mediante información numérica, gráficas y fórmulas.

El alcance de la investigación fue descriptivo - explicativo, ya que en la primera fase se realizó una descripción de las variables de estudio especificando sus características y propiedades, en la segunda fase, se explicó las causas que generaron problemas de logística en la empresa Megalimpio Cía. Ltda, considerada como unidad de análisis. Los métodos utilizados fueron: analítico - sintético, histórico - lógico e inductivo - deductivo, tanto para el tratamiento de la literatura como para el análisis de los resultados y la estructura de la propuesta. El universo de estudio estuvo conformado por 16 personas operativas y 5 administrativas, a quienes se les aplicó una encuesta estructurada con 48 ítems relacionados con el ambiente de control, evaluación de riesgo y actividades de control (incluido subcategorías de: entradas de inventario, almacenamiento, salidas, información y comunicación, supervisión y monitoreo).

\section{Resultados}

Los resultados de la aplicación de los instrumentos de investigación para el análisis de la gestión logística en Megalimpio Cía. Ltda. se muestran a continuación:

- Existe desconocimiento del código de ética y conducta, así como de la existencia de manuales, normas y procedimientos para el proceso de gestión de inventarios.

- No se ha identificado claramente los riesgos que inciden en el logro de los objetivos del proceso de gestión de inventarios de la empresa, imposibilitando que se ejecuten las acciones pertinentes para controlar adecuadamente los mismos.

- Existen falencias en el control de las entradas de inventarios, siendo necesario el replanteamiento de políticas y lineamientos. 
- La empresa no dispone de sistemas de seguridad suficientes que contribuyan a la salvaguarda de los inventarios, tales como: cámaras, extintores, plan de contingencia en caso de siniestro, controles de entrada y salida de materiales, siendo necesario el análisis del entorno para la implementación de mayores niveles de seguridad y ejecución de acciones preventivas.

- Las diferencias detectadas entre los registros y los resultados de la toma física no se analizan de forma crítica, para identificar los problemas que se presentan en el almacenamiento de los inventarios.

- Los registros contables no reflejan información fidedigna y actualizada respecto del saldo de inventarios, en base a las entradas y salidas efectuadas diariamente.

- Los canales de comunicación no se encuentran claramente establecidos, de forma que garanticen que los grupos de interés puedan reportar situaciones de fraude, asociados al proceso de gestión de inventarios.

\section{Propuesta.}

El sistema de gestión logística planteado para la empresa Megalimpio Cía. Ltda. se fundamenta en la planificación, control y evaluación de riesgos de la cadena de suministros, por lo que, permitirá contar con una provisión de insumos optima y un adecuado traslado del producto hasta la entrega a los clientes. En el proceso intervendrán seis subprocesos que están inmersos en la logística y que se enlazan a la normativa ISO 28000, control interno y costos.

Figura 2. Esquema de la propuesta sistema de gestión logística

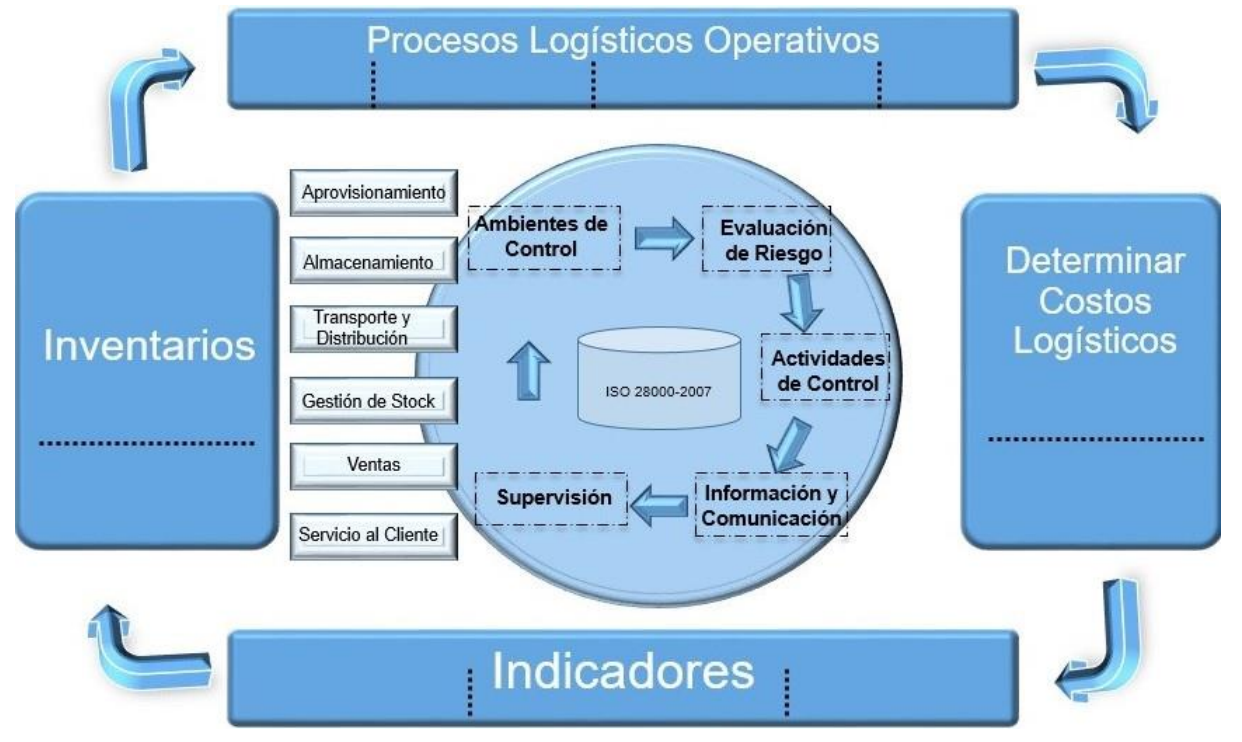


La responsabilidad de la dirección en cuanto al planteamiento de los componentes consiste en documentar el sistema de gestión de logística acorde a las fichas que se implementarán. La dirección para viabilizar la puesta en marcha del sistema estará a cargo del Gerente General, sus lineamientos estarán orientados al logro de metas y objetivos. La responsabilidad de la ejecución del sistema de gestión estará a cargo del jefe de logística, responsable de guiar la aplicación, difundir la información y los cambios que se realizan en la gestión de inventarios, su evaluación y control.

El planteamiento de los objetivos es esencial por ende se deben definir con claridad. De acuerdo con los componentes del sistema de gestión logístico se han planteado los objetivos de cada proceso, de manera tal, que se asegura su cumplimiento.

Todos los aspectos antes mencionados serán revisados para asegurar el cumplimiento de todos los requerimientos. La empresa al igual que el personal designado para esta labor de gestión deberán verificar el avance de los resultados acorde a la ideología planteada como parte del sistema.

\section{Compras y aprovisionamiento}

Objetivo: Establecer procedimientos de compras y aprovisionamiento que permitan eficiencia, efectividad, y control operativo y contable al proceso logístico de la empresa, otorgando valor a las compras y a la empresa en general.

Tabla 1. Compras y aprovisionamiento

\section{Compras}

- Recibir las solicitudes de compra de inventario, aprobados por la gerencia.

- Solicitar cotizaciones y seleccionar los proveedores, en el caso de que exista un requerimiento de producto nuevo.

- Gestionar los contratos de proveedores seleccionados que fueron aprobados en licitación que se encuentran en la base de datos.

- Informar al departamento de almacenamiento sobre todas las adquisiciones para su posterior distribución.

\section{Recepción}

- Verificar que la cantidad y calidad de productos este acorde a las necesidades y requerimientos. 
- Detectar si existen algún producto que sea defectuoso, dañado o que su defecto no esté en las condiciones requeridas.

- Revisar que lo entregado por los proveedores, este acorde a la factura y los comprobantes de ingreso.

- Se debe reportar diariamente los movimientos de inventarios.

\section{Entrega}

- La salida de la mercadería se realiza de acuerdo con el requerimiento de requisición la misma que está autorizada por el jefe inmediato.

\section{Pago a Proveedores}

- Corroborar que las facturas de los proveedores estén correctamente elaboradas y cumplan con los reglamentos y comprobantes.

- Verificar que las facturas concuerden con el contrato respectivo.

- Emitir el comprobante de egreso y la transferencia respectiva.

\section{Políticas}

- Definir los productos que se requieren para tipo de negocio que se va a realizar.

- Mantener parámetros de calidad en los productos que se distribuye.

- Mantener la continuidad de los productos para evitar inconsistencias en la información.

- Conocer las tendencias de los consumidores y necesidades en función de las actividades que se van a realizar con relación a la limpieza.

- Análisis y aceptación de los proveedores que cuenten con productos amigables para el medio ambiente.

\section{Responsabilidad}

- Jefe de compras

Cierre de reportes contables

\begin{tabular}{|l|l|l|c|c|}
\hline Fecha & \multicolumn{1}{|c|}{ Cuenta } & \multicolumn{1}{|c|}{ Descripción } & Debe & Haber \\
\hline & $\begin{array}{l}\text { Compras Trans. Fuente: PE No. 12804 / } \\
\text { Inventario de mercadería }\end{array}$ & $\begin{array}{l}\text { Pedido } \\
\text { Compras Trans. Fuente: PE No. 12804/ } \\
\text { Pedido }\end{array}$ & & \\
\hline & $\begin{array}{l}\text { Iva pagado en compras } \\
\text { Proveedores por pagar }\end{array}$ & $\begin{array}{l}\text { Compras Trans. Fuente: PE No. 12804 / } \\
\text { Pedido }\end{array}$ & & \\
\hline
\end{tabular}

\section{Controles}

- Archivo de la documentación existente.

- Cruce de información con documentos soporte y registros. 


\section{Almacenamiento}

Objetivo: Establecer la gestión de almacenamiento que admita garantizar la seguridad del stock bajo esquemas de eficiencia y optimización de costos.

Tabla 2. Almacenamiento

\section{Recepción de pedidos.}

- Introducción de los pedidos al sistema.

- Inspección del estado de los productos y los procedimientos de seguridad.

- Establecimiento del inventario al almacén en el sistema.

- Realizar un muestreo de aceptación del producto verificación de las condiciones de calidad para recudir los reclamos.

\section{Movimiento y ubicación.}

- Establecer la distribución adecuada de cada uno de los productos considerando especificaciones de seguridad del producto.

- Colocar los productos de acuerdo con la especificación de las estanterías.

\section{Políticas}

- En el almacenamiento de los productos está prohibido fumar ya que gran parte de los productos son inflamables.

- En el manejo y movimiento de los productos debe acreditarse mediante reglamentaciones internas

- Todos los productos deberán ser codificados.

\section{Responsabilidad}

- Jefe de almacenamiento

- Asistente de bodega.

\section{Cierre de reportes contables}

Compra Nro.

\section{Megalimpio Cía. Ltda.}

Fecha de Ingreso:

Proveedor:

\begin{tabular}{|c|c|c|}
\hline Cod. Ítem & Descripción & Cantidad \\
\hline & & \\
\hline
\end{tabular}

\section{Controles}

- Archivo de la documentación existente

- Cruce de información con compras. 


\section{Transporte y distribución}

Objetivo: Establecer el procedimiento de transporte y distribución de los productos considerando la eficiencia, registro, control, mantenimiento y optimización del uso de recursos.

Tabla 3. Transporte y distribución

\section{Asignación de transporte}

- El chofer será responsable de la unidad de transporte,

- Mantendrán un registro de las actividades y responsables del transporte de los productos.

- Se establece un acta de entrega del vehículo y la custodia de este.

- Serán responsables del mantenimiento preventivo y correctivo del vehículo.

\section{Transporte}

- Establecer una estrategia de transporte apropiada para la optimización y reducción de costos

- Garantizar una amplia alternativa de servicio de acuerdo con la capacidad instalada de la empresa

- Considera la modalidad, tamaño y los gastos de envíos, con la implicación del servicio

- Mantener indicadores de gestión como parte de del desempeño de transporte.

- Los tipos de carga estarán designados de acuerdo con la cantidad de los productos.

\section{Distribución}

- Establecer el canal adecuado para la distribución del producto.

- Brindar una cobertura eficiente de los productos por los diversos canales.

- Mantener altos estándares de calidad durante el proceso de distribución.

- Considerar los costos en la distribución del producto.

- Estrategias para establecer la distribución del producto.

\section{Políticas}

- Todos los productos serán distribuidos después de un día hábil.

- En el transporte y distribución de los productos se realizará mediante guía de remisión.

- Los controles de transporte se realizan siempre para evitar el uso inadecuado de las unidades.

\section{Responsables}

- Chofer 


\section{Gestión de stock}

Objetivo: Establecer los procedimientos de gestión y documentación de los productos almacenados con el fin de optimizar costos y mantener el control.

Tabla 4. Gestión de stock

\section{Recepción de compras}

- Las compras que se reciban conforme a los pedidos deberán ser ingresados a la bodega con sus correspondientes documentos de ingreso de tal manera que los kárdex se encuentren actualizados.

\section{Devoluciones}

- Los inventarios que sean objeto de devoluciones serán despachados con todos los documentos de descargo, antes del ingreso de los productos al sistema.

- La verificación en el proceso de recepción es indispensable.

- En caso de que por algún motivo no puedan ser despachados/entregados los productos serán segregados y puestos un mensaje "NO CONTAR".

\section{Despachos}

- Los despachos se realizarán conforme a los pedidos que se registran en el día.

\section{Políticas}

- Aprovisionamiento de todos los productos que tienen una alta demanda.

- Contar con un inventario que esté acorde a las ventas que se registran en los últimos años.

- Minimizar la inversión en stock

- Establecer información eficiente para la venta de productos de limpieza.

\section{Responsables}

- Jefe de compras

- Bodeguero

- Jefe de Almacén

- Chofer

Para una óptima gestión de stock se hará uso de la cantidad económica de pedido, haciendo uso de la información de la empresa, para realizar pedidos, especialmente del inventario con mayor requerimiento mediante la siguiente fórmula:

$$
\text { Cantidad económica de pedido }=\sqrt{\frac{2(\text { Demanda })(\text { Costo de pedido })}{\text { Costo de mantener inventario }}}
$$




\section{Ventas y servicio al cliente}

Objetivo: Garantizar la eficiencia, seguridad, control de las ventas con enfoque en la satisfacción del cliente tanto en la entrega de servicios de calidad, como una óptima atención al cliente.

\section{Tabla 5. Ventas}

\section{Atención al cliente}

- Recibir llamadas telefónicas de clientes recurrentes y de posibles clientes y elaborar un reporte de requerimientos solicitados.

- Satisfacer las inquietudes del cliente, cuando solicite una proforma de precios de los servicios que ofrece la empresa.

- Atender a los clientes que acuden a las instalaciones para adquirir los productos

- Coordinar la visita personal a los clientes contactados y elaborar un reporte de requerimientos solicitados.

\section{Facturación de productos}

- Corresponde a la facturación de los productos; en él se definen la cantidad, descripción, precios y total.

- Los clientes deben registrarse en la base de datos.

- Verificación de los productos y la disponibilidad en stock.

- Emitir las facturas con información de los clientes y sus respectivos requerimientos.

\section{Entrega}

- Entrega de los productos de acuerdo con las especificaciones solicitadas por el cliente.

- Gestionar el pago de la factura con clientes que no tengan asignado crédito.

\section{Políticas}

- Las promociones y descuentos deberán respetarse en los meses y fechas preestablecidas por el departamento.

- Las ventas no se realizarán sin previa verificación de stock.

- No se aceptan devoluciones ni cambios salida la mercadería.

- El vendedor debe explicar claramente de los productos e insumos que adquirirá el cliente.

\section{Responsables}

- Jefe de ventas, Servicio al cliente y facturación.

\begin{tabular}{|c|c|c|c|c|}
\hline \multicolumn{5}{|c|}{ Cierre de reportes contables } \\
\hline Fecha & Cuenta & Descripción & Debe & Haber \\
\hline & Cuentas Por Cobrar Clientes & Factura Almacén & & \\
\hline & Costo De Ventas Almacén & Factura Almacén & & \\
\hline & Inventario de Mercadería & Factura Almacén & & \\
\hline & Iva Cobrado En Ventas & Factura Almacén & & \\
\hline & Ventas De Mercadería $12 \%$ & Factura Almacén & & \\
\hline
\end{tabular}


Responsable

- Asistente de facturación

- Contador

\section{Controles}

- Secuencial asignado automáticamente

Establecidos los procedimientos es necesario establecer los principios y registros con los que dará soporte al control, a una mejor gestión de costos y a la minimización de los riesgos.

\section{Ambiente de control}

Para mejorar el ambiente de control, además de lo considerado en los procedimientos, se establecen parámetros de control interno dentro de los cuales se hará uso de cuestionarios para la verificación del cumplimiento, parámetros que serán modificados con el tiempo, manteniendo principios de mejoramiento continuo. Entre las principales preguntas están:

Tabla 6. Cuestionario de control

\begin{tabular}{|c|c|c|c|}
\hline Pregunta & $\begin{array}{ll}\text { Si } & \text { No }\end{array}$ & Parcial & Observaciones \\
\hline $\begin{array}{l}\text { - ¿Se hace validación periódica de las salidas de almacén y los } \\
\text { registros en los sistemas de información? } \\
\text { ¿Los inventarios están registrados y valorados a valor de } \\
\text { mercado? } \\
\text { - ¿Se tiene un control consecutivo de salidas de almacén para retiros } \\
\text { de productos? } \\
\text { - ¿Se deja evidencia de los resultados de las tomas físicas? } \\
\text { ¿Se analizan e investigan las diferencias entre los registros y los } \\
\text { - ¿Sesultados de las tomas físicas? } \\
\text { ¿Se investigan y analizan frecuentemente roturas, desperdicios y } \\
\text { bajas de producto? } \\
\text { ¿Los depósitos y bodegas cuentan con adecuados sistemas de } \\
\text { seguridad para la salvaguarda del inventario (cámaras, extintores, } \\
\text { plan de contingencia, controles de entrada y salida ...)? } \\
\text { ¿Se dispone de locaciones y ubicaciones de productos } \\
\text { debidamente ordenados y estratégicamente dispuestos para } \\
\text { asegurar la gestión de primeros en entrar primeros en salir - PEPS? }\end{array}$ & & & \\
\hline
\end{tabular}




\section{Políticas:}

- Se implementará un comité de auditoría que permita asegurar la integridad de todos los procedimientos.

- Se dispondrá de reglamentos internos socializados y al alcance del personal en donde se explica con claridad la filosofía, reglamentos, roles, procedimientos y responsabilidades a cumplir.

- Los medios de comunicación a utilizarse serán: software integrado de inventarios, uso de chat interno para una comunicación rápida y eficaz.

- Establecer estándares de desempeño y cotejar los resultados con los estándares establecidos

- Si existiesen variaciones, es necesario determinar las causas y efectuar las acciones correctivas.

\section{Supervisión}

- Realizar el seguimiento del proceso actual.

- Se establecerá un encargado de la supervisión del programa, los procesos, actividades de control y el registro de indicadores.

- La supervisión se realizará diariamente en las áreas de descarga, bodega, entrega de inventario para el servicio.

- Se establecerá anualmente el cronograma de acciones para control y operación de la gestión logística.

- Se realizará auditorías aleatorias en medición de cantidad y servicio.

- Parámetros de verificación: Cantidad, peso, estado de inventario, autorización de recepción y despacho, registros y documentos de respaldo.

- Establecer firma de responsabilidad de operario y supervisor.

\section{Evaluación de riesgos}

Para la evaluación de riesgos en el sistema logístico se partirá del planteamiento de un esquema que será aplicado a la empresa para determinar el impacto de los riesgos. 
Tabla 7. Registro y evaluación de riesgos

\section{Empresa Megalimpio Cía. Ltda.}

\begin{tabular}{|c|c|c|c|c|c|c|}
\hline \multicolumn{7}{|l|}{ Responsable } \\
\hline \multicolumn{7}{|c|}{ Evaluación de riesgos } \\
\hline COMPONENTES & Criterios & Impacto & Mitigación & Monitoreo & $\begin{array}{c}\text { Nivel de } \\
\text { riesgo }\end{array}$ & $\begin{array}{c}\text { Partes } \\
\text { involucradas }\end{array}$ \\
\hline \multicolumn{7}{|l|}{ Aprovisionamiento } \\
\hline \multicolumn{7}{|l|}{ Gestión de Stock } \\
\hline \multicolumn{7}{|l|}{ Recomendaciones } \\
\hline Observaciones & & & & & & \\
\hline
\end{tabular}

Para dar seguimiento a los riesgos se utiliza la matriz como un instrumento se control de las actividades y procesos en este sentido se manejará de la misma manera un formato específico para el tratamiento del riesgo.

Tabla 8. Matriz de análisis de riesgos

\begin{tabular}{|c|c|}
\hline \multicolumn{2}{|r|}{ Empresa Megalimpio Cía. Ltda. } \\
\hline \multicolumn{2}{|r|}{ Matriz de Riesgo } \\
\hline \multicolumn{2}{|l|}{ Proceso } \\
\hline \multicolumn{2}{|l|}{ Objetivo } \\
\hline \multicolumn{2}{|c|}{ Identificación de riesgo } \\
\hline Causa & Riesgos \\
\hline Consecuencia & Ocurrencia \\
\hline \multicolumn{2}{|r|}{ Niveles de consecuencia } \\
\hline \multicolumn{2}{|c|}{ Nivel de riesgo $=$ Probabilidad de ocurrencia $\times$ Impacto $/ 100$} \\
\hline & Análisis de riesgo \\
\hline
\end{tabular}

\section{Responsable}

\section{Observaciones}

\section{Indicadores de control}

Se han considerado los principales indicadores de control en el área de abastecimiento, ventas, facturación y servicio. 
Tabla 9. Indicadores de control

Cumplimiento de plazos $(\%)=\frac{\text { Número de pedidos recibidos en el plazo previsto }}{\text { Número total de pedidos recibidos }} \times 100$

Volumen de compras $=\frac{\text { Valor de compras }}{\text { Total de ventas }}$

Participación del costo de almacenamiento $=\frac{\text { Costo de almacenamiento }}{\text { Total de ventas }} \times 100$

Errores de previsión de demanda $=\frac{\text { Previsión de la demanda }- \text { Demanda real }}{\text { Remanda Real }}$

Plazo de aprovisionamiento (Lead Time)

$=$ Fecha recepción pedido - Fecha emisión pedido

Plazo medio de pago $=\frac{\sum \text { Número de días pasados desde la emisión de factura hasta el pago }}{\text { Número total de facturas }}$

Entrega de servicio perfecto $=\frac{\text { Entrega de servicio perfecto }}{\text { Total de entregas de servicio }}$

\section{Conclusiones.}

- Los modelos de gestión de inventarios integran las herramientas y técnicas que permiten la determinación de los costos y la mitigación de riesgos en el manejo de la mercancía, por lo que, se hace necesario la planificación y el establecimiento de políticas y objetivos a corto mediano y largo plazo con la finalidad de incrementar la rentabilidad de la empresa, en lo concerniente al manejo de los productos.

- Toda empresa requiere de un sistema de información que contribuya a medir las diferentes etapas del procedimiento logístico, y para esto se tienen varios grupos de indicadores como son costos, financieros, productividad, estos ayudarán a ser más competitivos y tomar los correctivos que la empresa deberá emplear en su momento.

- La investigación en la empresa Megalimpio Cía. Ltda., ha permitido determinar que existen procesos, documentación e inclusive ciertos principios que guían la administración y el control de los inventarios, sin embargo, no está institucionalizado como política de la empresa y por tanto no todos los empleados conocen y lo aplican, 
lo cual ha llevado a la necesidad de implementar un sistema para el control de los inventarios de toda la cadena de suministro y disminuir los riesgos que pueda tener, especialmente en este caso para una empresa comercializadora en la que tiene alta importancia la gestión de stock.

- Se cuenta con una norma internacional como es la ISO 28000 que es un sistema de gestión que da seguridad a la cadena de suministros y está orientada a buscar elementos de control para programar la seguridad, lo que permite valorar los riesgos inherentes a las actividades, apegándose a requisitos legales y reglamentos de seguridad internacionales, la cual ha sido utilizada como soporte a la gestión de inventarios de la empresa Megalimpio Cía. Ltda.

\section{Referencias bibliográficas.}

Anaya, J. (2007). Logística integral: la gestión operativa de la empresa. Madrid, España: ESIC.

Ballau, R. (2004). Logística Administración de la cadena de suministro. Mexico, Mexico: Pearson Educación.

Casanovas, A. y Cuatrecasas, L. (2012). Logística integral. Barcelona: Profit Editorial. Obtenido de https://books.google.com.ec/books?id=68tx0owyJ0wC

Escudero, M. (2011). Gestión de aprovisionamiento: administración. España: Edisiones Paraninfo. Obtenido https://books.google.es/books?id=jabS4x3L2oEC\&lpg=PR1\&ots=2bEJPzDXcz\& $\mathrm{dq}=\mathrm{La} \% 20 \mathrm{gesti} \% \mathrm{C} 3 \% \mathrm{~B} 3 \mathrm{n} \% 20 \mathrm{de} \% 20 \mathrm{las} \% 20$ existencias\&lr\&hl=es\&pg=PA161\# $\mathrm{v}=$ onepage $\& \mathrm{q}=$ gesti $\% \mathrm{C} 3 \% \mathrm{~B} 3 \mathrm{n} \& \mathrm{f}=$ false

Gibson, B. Mentzer, J y Cook, R (2005). Supply chain management: the pursuit of a consensus definition. Journal of Business Logistics. Vol. 26, pp. 17-25.

González, J. (2002). Control y gestión del área comercial y de producción de la PYME. Coruña: Netbiblo.

Gutiérrez, G. y Prida, B. (1998). Logística y distribución física. Madrid: McGraw-Hill Interamericana.

Horngren, C., Sundem, G., y Stratton, W. (2007). Contabilidad administrativa. Pearson Educación. Obtenido de 
https://books.google.com.ec/books?id=fRi9LKlr03UC\&lpg=PP1\&pg=PP1\#v=one page \&q\&f=false

Larrucea, J. (2015). Seguridad marítima: Teoría general del riesgo. Barcelona: Marge Books. Obtenido https://books.google.com.ec/books?id=jH89CgAAQBAJ\&pg=PA161\&dq=Objeti vo+de+la+norma+ISO+28000\&hl=es419\&sa=X\&ved=0ahUKEwjhwvSss53aAhVP7VMKHQT0A0AQ6AEIJjAA\#v= onepage $\& q \& \mathrm{f}=$ false

Manzur, N. (2014). La mercadotecnia y los pasos de su éxito. Editamex afiliado a asociación de autores y editores. Obtenido de https://books.google.com.ec/books?id=ahzxAwAAQBAJ

Mora, L. (2016). Gestión Logistica Integral. Las mejores prácticas en la cadena de abastecimiento. (Segunda ed.). Bogotá: ECOE Ediciones.

Mora, L. y Martiliano, M. (2010). Modelos de optimización de la gestión logística. Bogota: ECOE Ediciones.

Perez. (2006). Modelo y procedimientos para el Control de Gestión. Cuba: Holguín.

Servera, D. (2010). Concepto y evolución de la función logística. INNOVAR. Revista de Ciencias Administrativas y Sociales, 19 . Obtenido de http://www.redalyc.org/articulo.oa?id=81819024018

SCP Supply Chain Professionals. (03 de 04 de 2018). Scpconsulting.net. Obtenido de http://www.scpconsulting.net/seguridad-en-cadena-de-suministros.html

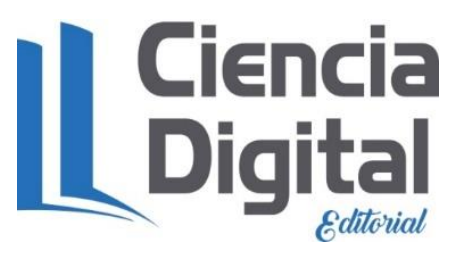




\section{PARA CITAR EL ARTÍCULO INDEXADO.}

Rocano Criollo, D., Narváez Zurita, C., Erazo Álvarez, J., \& Luna Altamirano, K. (2019). Gestión logística con enfoque en la ISO28000, para empresas comerciales. Visionario Digital, 3(2.1.), 136-158. https://doi.org/10.33262/visionariodigital.v3i2.1.550

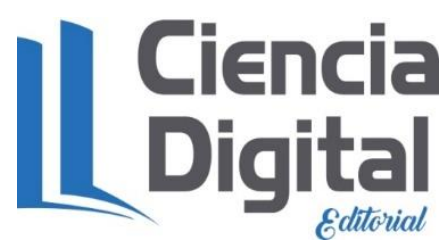

El artículo que se publica es de exclusiva responsabilidad de los autores y no necesariamente reflejan el pensamiento de la Revista Ciencia Digital.

El artículo queda en propiedad de la revista y, por tanto, su publicación parcial y/o total en otro medio tiene que ser autorizado por el director de la Revista Ciencia Digital.
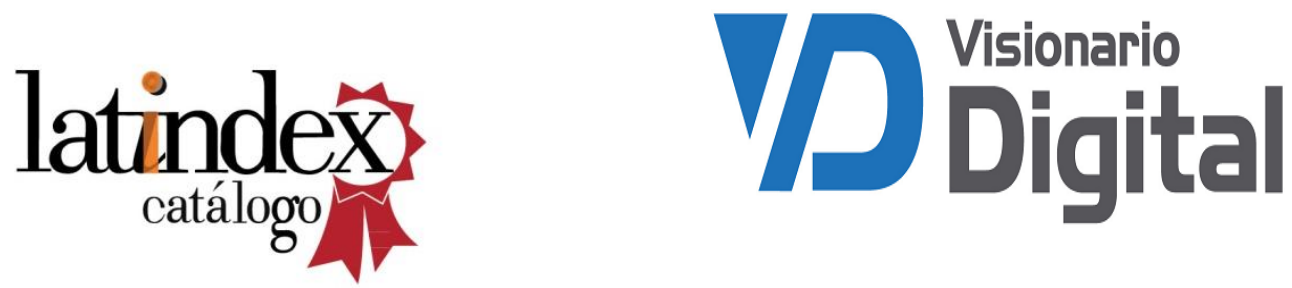